\title{
INFINITE COXETER GROUPS ARE VIRTUALLY INDICABLE
}

\author{
by D. COOPER, D. D. LONG and A. W. REID*
}

(Received 5th July 1996)

We prove that any infinite Coxeter group has a finite index subgroup which surjects $\mathbb{Z}$.

1991 Mathematics subject classification: 20 F55.

\section{Introduction}

An infinite group $G$ is called indicable (resp. virtually indicable) if $G$ (resp. a subgroup of finite index in $G$ ) admits a homomorphism onto $\mathbb{Z}$. This is a powerful property for a group to have; for example in the context of infinite fundamental groups of aspherical 3-manifolds it remains one of the outstanding open questions to prove such groups are virtually indicable. To continue on the 3-manifold theme, it follows from the work of Hempel [8] that any closed orientable hyperbolic 3-manifold which admits an orientation-reversing involution has fundamental group that is virtually indicable. In particular if a closed hyperbolic 3-manifold $M$ is a finite cover of a hyperbolic 3orbifold obtained as the quotient of $\mathbb{H}^{3}$ by a group generated by reflections (i.e., a hyperbolic Coxeter group) then $\pi_{1}(M)$ is virtually indicable.

The purpose of this note is to prove the following theorem, posed as a question by P. De La Harpe and A. Valette ([5]) in connection with Property T (see below):

Theorem 1.1. Let $W$ be an infinite Coxeter group, then $W$ is virtually indicable.

Our methods are motivated from those of low-dimensional topology, in particular the work in [9] and [10] which deal with "separability properties" of 3-manifold groups.

This theorem has several consequences which seem independently interesting. For example it implies:

Corollary 1.2. Let $W$ be an infinite Coxeter group and $K$ any subgroup of finite index in $W$. Then $K$ is not isomorphic to an irreducible lattice in a semisimple Lie group of $\mathbb{R}$ rank $\geq 2$.

- The first two authors are supported in part by the NSF and the third by the NSF and The Royal Society. The first and third authors also wish to thank the Institut Henri Poincare for its hospitality whilst completing this work. 
The corollary follows directly from Theorem 1.1, since the work of Margulis (see [12]) shows that any irreducible lattice in such a Lie group is necessarily arithmetic and any normal subgroup is either finite or finite index. In fact one can say a little more.

Corollary 1.3. Let $W$ be an infinite Coxeter group and $K$ any subgroup of finite index in $W$. Then $K$ does not satisfy Kazhdan's Property $T$.

This was first proved in [3] in a little more generality. This corollary also implies that an infinite Coxeter group cannot be a uniform lattice in the rank 1 Lie groups, $\operatorname{Sp}(n, 1)$ or $\mathbb{F}_{4}$. Thus the only $\mathbb{R}$-rank 1 Lie groups for which an infinite Coxeter group can be a lattice are $\operatorname{SO}(n, 1)$ or $\operatorname{SU}(n, 1)$. Mike Davis has pointed out to us that it follows from work of his [4] that an infinite Coxeter group cannot be an irreducible lattice in $\operatorname{SU}(\mathbf{n}, 1)$.

We now outline our method of proof deferring definitions to the body of the text. Let $W$ be the Coxeter group. Coxeter groups act on a canonical simply connected complex, the Coxeter complex, and the strategy is to show that this complex contains a subcomplex which behaves like a totally geodesic codimension one submanifold in hyperbolic space. This is accomplished in two steps.

The Coxeter complex is equipped with certain codimension one subcomplexes called walls. The first step is to show that one can find some wall so that its stabiliser, $H$, is a separable subgroup of infinite index in $W$. Then, motivated by standard techniques coming from 3-manifolds, (for example see [9]) we show that one can pass to a subgroup of finite index in $W$, so that the quotient of the Coxeter complex by this subgroup contains an embedded nonseparating codimension one subcomplex, namely the image of the chosen wall. This gives a map onto the integers.

Although not used in the proof, it seems to be of some interest that we may also show:

Theorem 1.4. Let $K$ be any special subgroup of the Coxeter group $W$. Then $K$ is separable in $W$.

Finally we remark that it would seem likely (as in the case of reflection groups acting on hyperbolic 3-space) that an infinite Coxeter group that is not virtually abelian has a finite index subgroup that maps onto a free group of rank at least two. We leave this as a question for the present.

We have been informed by Mike Davis that Theorem 1.1 has been proved independently and by different methods by Constantin Gonciulea and will appear in his Ohio State University Ph.D Thesis, see also [6].

\section{Coxeter groups and the Coxeter complex}

We recall some salient points about Coxeter groups and the associated Coxeter Complex that we shall require, see [2] for details. 
2.1.

Definition 2.1. Suppose that $W$ is a group and $S$ is a set of generators all of order 2. Then $(W, S)$ is a Coxeter system if $W$ admits a presentation:

$$
\left\langle S \mid(s \cdot t)^{m(s, t)}=1\right\rangle
$$

where $m(s, t)$ is the order of $s \cdot t$ and there is one relation for each pair $s, t$ with $m(s, t)<\infty$.

We often abuse this notation and refer to $W$ as a Coxeter group. Many equivalent definitions exist, see [2]. The Coxeter diagram of this presentation consists of a vertex for each element of $S$ together with an edge connecting distinct vertices $s, t$ whenever $m(s, t) \neq 2$ and the edge is labelled by $m(s, t)$. Since the generators have order 2, this means that if two vertices are not connected by an edge then the generators corresponding to the vertices commute. Thus, if the diagram is not connected, the Coxeter group is the direct sum of the subgroups given by the connected components. A Coxeter group $(W, S)$ is called reducible if its diagram is not connected.

2.2. Next we recall the construction of the Coxeter complex $\Sigma$ of the Coxeter system $(W, S)$. Firstly, by a special subgroup of $W$ we mean a subgroup $\left\langle S^{\prime}\right\rangle$ of $W$ where $S^{\prime} \subset S$. There is a simplicial complex $\Sigma=\Sigma(W, S)$ specified as follows (see [2, p. 33]). If $n=|S|$ and $k<n$, the $k$-simplices of $\Sigma$ are the left-cosets of special subgroups generated by $n-1-k$ distinct elements of $S$. Thus if $S^{\prime}$ is a subset containing $n-1-k$ elements of $S$ and $w$ is any element of $W$ then $w \cdot\left\langle S^{\prime}\right\rangle$ is a $k$-simplex. The incidence relation between simplices corresponds to the containment relation between cosets. In particular, the $(n-1)$-simplices correspond to the elements of $W$ and the dual 1skeleton of $\Sigma$ is a modified Cayley graph of $W$ with generating set $S$. The modification consists of identifying the edge labelled $s$ with the edge labelled $s^{-1}$ for each generator $s \in S$. The action of $W$ on the left cosets by left multiplication induces a simplicial action of $W$ on $\Sigma$. A top dimensional simplex, $C$, of $\Sigma$ is called a chamber. Observe that the only element of $W$ which maps some chamber to itself is the identity (see [2, Chapter III, \$4 Lemma 6]).

Following [2, (Chapter III, §4)], we see that given any pair of adjacent chambers $C$ and $C^{\prime}$, there is a unique automorphism $s$ of the Coxeter complex of order 2 which exchanges $C$ and $C^{\prime}$ while fixing $C \cap C^{\prime}$, and this gives rise to a wall (denoted by $\mathcal{H}_{s}$ ) in the Coxeter complex, namely $\mathcal{H}_{s}=F i x(s)$. Conversely, any reflection in $W$ gives rise to a unique wall. Note that walls separate the Coxeter complex. (See [2], for example, Chapter III §3 Corollary 3.)

Fix the following notation: let $g \in W$, then $C_{W}(g)$ denotes the centralizer of $g$ in $W$. 
Lemma 2.2. $s t a b\left(\mathcal{H}_{s}\right)=C_{W}(s)$

Proof. Let $\gamma$ be an element of $\operatorname{stab}\left(\mathcal{H}_{s}\right)$. Then $\gamma\left(C \cap C^{\prime}\right)$ is some codimension-1 face in $\mathcal{H}_{s}$ and is therefore fixed by $s$. It follows that $s$ and $\gamma^{-1} \cdot s \cdot \gamma$ are both automorphisms of order two fixing $C \cap C^{\prime}$ pointwise and exchanging $\gamma C$ with $\gamma C^{\prime}$. Thus $\gamma^{-1} \cdot s \cdot \gamma \cdot s^{-1}$ maps $C$ to itself and therefore is the identity element of $W$. (See [2, Chapter III, $\S 4$ Lemma 6].)

Conversely, if $\gamma \in C_{W}(s)$, then $\gamma^{-1} \cdot s \cdot \gamma \cdot s^{-1}$ is the identity. It follows that $s\left(\gamma \mathcal{H}_{s}\right)=\gamma \mathcal{H}_{s}$. As $s$ fixes a unique wall, we deduce that $\gamma \mathcal{H}_{s}=\mathcal{H}_{s}$, and $\gamma \in \operatorname{stab}\left(\mathcal{H}_{s}\right)$ as was required.

2.3. In the remainder of this section we fix attention on the case where all 2generator special subgroups are finite dihedral as this will be important in what follows. That this suffices for our application will be proven in Lemma 3.6.

Note in particular that this restriction implies that every codimension- 2 simplex of the Coxeter complex $\Sigma$ is the face of only finitely many codimension-1 simplices.

Consider the dual 2-complex $X$ of $\Sigma$. This has a vertex for each chamber and two vertices are connected by an edge if the corresponding chambers are adjacent along a codimension-1 face. Chambers have a finite number, $|S|$, of codimension one faces, so this gives a 1-complex with each vertex of degree $|S|$. Each edge in this complex corresponds to a coset of a special subgroup $\langle s\rangle$ for some reflection $s \in S$. There is a 2 cell for each codimension-2 simplex in $\Sigma$; these correspond to cosets of special subgroups of the form $\langle r, s\rangle$ which in the case at hand case are finite dihedral groups, so that each such 2-cell has a finite number of sides given by the cardinality of this dihedral group.

The complex $X$ is closely related to another complex $\tilde{Y}$ which we now describe. The presentation of the Coxeter system $(W, S)$ gives rise, in the usual way, to a 2-dimensional $\mathrm{CW}$-complex with fundamental group $W$. Thus $Y$ has one vertex, and an edge for each element of the generating set $S$. It has one 2-cell for each of the relations in the Coxeter presentation. Thus there are 2-cells for the relation of the form $s^{2}=1$ and for relations of the form $(s \cdot t)^{m(s, t)}=1$ when $m(s, t)<\infty$. Then $W$ acts freely on the universal cover, $\tilde{Y}$ of $Y$. Now $X$ is obtained as a quotient of $\tilde{Y}$ by collapsing to a 1-cell each 2-cell covering a relation of the form $s^{2}=1$. To be more specific, each edge, $E$, of $Y$ has a 2 -cell, $A$, attached by a degree- 2 map. This is covered in $\tilde{Y}$ by a 2 -sphere, $B$, and the covering transformation corresponding to $E$ acts as the antipodal map on $B$. Now $B$ consists of two lifts of $E$ and two lifts of $A$. Retract $B$ to one of the lifts of $E$. Doing this for every edge of $\tilde{Y}$ gives $X$. In particular, since $Y$ is finite, it follows that $\tilde{Y}$ and $X$ are both locally finite. Since $\tilde{Y}$ is simply connected, and $X$ is a retract of $\tilde{Y}$, it also follows that $X$ is simply connected.

There is an obvious action of $W$ on the complex $X$. The quotient is a complex of groups in the sense of Haefliger (see [7]) and the underlying group of this complex of groups is $\pi_{1}^{\text {orb }}(X / W) \cong W$. In fact, using that $W$ is a linear group, we will pass to a 
torsion-free subgroup of finite index in $W$ and this subgroup acts freely on $X$. This means we may deal with topological fundamental group in place of $\pi_{1}^{\text {orb }}$.

There is an obvious embedding $X \rightarrow \Sigma$ which is $W$-equivariant and so that the action of $W$ on $\Sigma$ restricts to the above action of $W$ on $X$.

\section{Proofs}

We recall that a subgroup $H$ of a group $G$ is said to be separable in $G$, if given any element $g \in G$ not lying in $H$, there is a subgroup $K$ of finite index in $G$ with the property that $H \leq K$ and $g$ does not lie in $K$. A group for which the trivial subgroup is separable is said to be residually finite. If a group has separable subgroups this often proves to be a powerful tool in questions concerning the homology of subgroups of finite index, see [9] and [10] for details.

3.1. Proving subgroups are separable seems to be rather difficult in general. The starting point of this work is the following result of [10]. For the convenience of the reader we include a proof:

Theorem 3.1. [10]. Let $\alpha: G \rightarrow G$ be an automorphism of a residually finite group G. Then Fix $(\alpha)$ is separable in $G$.

Proof. Choose an element $\gamma$ not lying in Fix $(\alpha)$. This means that the element $\gamma^{-1} \cdot \alpha(\gamma)$ is not the identity element, so that there is a homomorphism $\phi: G \rightarrow F$ onto a finite group $F$, so that $\phi\left(\gamma^{-1} \cdot \alpha(\gamma)\right)$ is not the identity element. Define a homomorphism

$$
\Phi: G \rightarrow F \times F
$$

by $\boldsymbol{\Phi}(g)=(\phi(g), \phi(\alpha(g)))$. Note that $\Phi$ maps Fix $(\alpha)$ into the diagonal subgroup of $F \times F$, however by construction, $\boldsymbol{\Phi}(\gamma)=(\phi(\gamma), \phi(\alpha(\gamma)))$ does not lie in the diagonal subgroup, so that $\Phi^{-1}\{(f, f) \mid f \in F\}$ is the required subgroup of finite index.

It is a theorem of Tits (see [2, Theorem C, p. 55]) that Coxeter groups have a faithful representation into some $G L(n, \mathbb{R})$, in particular this means Coxeter groups are linear, and hence are residually finite. Noting that the centralizer of a generator, $s$, of $W$ is the fixed set of the automorphism $W \rightarrow W$ given by $g \rightarrow s \cdot g \cdot s$, Theorem 3.1 shows:

Corollary 3.2. $\quad C_{W}(s)$ is a separable subgroup of $W$.

This together with Lemma 2.2 then implies,

Corollary 3.3. In the notation above stabH, is a separable subgroup of $W$. 
3.2. Recall that a subgroup $K \leq W$ is a special subgroup if it is generated by some subset of $S$. The following is not used in the proof of the main theorem, but is of some interest:

Theorem 3.4. Let $K$ be any special subgroup of the Coxeter group $W$. Then $K$ is separable in $W$.

Proof. By reordering the elements of $S=\left\{s_{1}, \ldots, s_{n}\right\}$, we may suppose that $K$ is generated by $\left\{s_{k+1}, \ldots, s_{n}\right\}$. We form the amalgamated free product group $G=W *_{K} W$ and use the notation $W_{L}, W_{R}$ for the two subgroups of $G$ which are the copies of $W$.

Our first claim is that $G$ is a Coxeter group. To this end, we note that by standard properties of free products with amalgamations, $G$ has a presentation with generators

$$
S_{G}=\left\{s_{1}^{L}, \ldots, s_{k}^{L}, s_{1}^{R}, \ldots, s_{k}^{R}, s_{k+1}, \ldots, s_{n}\right\}
$$

and relations of the form

$$
\begin{aligned}
& \left(s_{i}^{L} \cdot s_{j}^{L}\right)^{m(i, j)}=1 \quad 1 \leq i, j \leq k \\
& \left(s_{i}^{R} \cdot s_{j}^{R}\right)^{m(i, j)}=1 \quad 1 \leq i, j \leq k \\
& \left(s_{i}^{L} \cdot s_{j}\right)^{m(i, j)}=1 \quad 1 \leq i \leq k \quad k+1 \leq j \leq n \\
& \left(s_{i}^{R} \cdot s_{j}\right)^{m(i, j)}=1 \quad 1 \leq i \leq k \quad k+1 \leq j \leq n .
\end{aligned}
$$

If $s, t$ are generators in $S_{G}$ define $m_{G}(s, t)$ to be the appropriate $m(i, j)$ from the above presentation, or else $\infty$. There is a homomorphism $G \rightarrow W$ defined on the generators by forgetting the $L-R$ labels. Using this, and properties of the normal form for words in an amalgamated free product, we see that given two elements $s, t$ in $S_{G}$ then the order of $s \cdot t$ is precisely $m_{G}(s, t)$.

Since $G$ is a Coxeter group, as remarked above, it is residually finite. Moreover there is an automorphism $\tau: G \rightarrow G$ of order two, which comes by using the obvious map to exchange $W_{L}$ and $W_{R}$. Again, using properties of the normal form, we see that the fixed subgroup of $\tau$ is precisely the amalgamating subgroup, a group which is isomorphic to $K$. Then Theorem 3.1 shows that $K$ is separable in $G$. It follows that $K$ is separable in $W_{L} \cong W$.

3.3. We now collect some facts that will be used in the proof of Theorem 1.1.

Lemma 3.5. Let $G$ be an infinite group generated by a finite set $S$. Then either $G$ has a free abelian subgroup of finite index or for some $g$ in $S$ the centralizer $C(g)$ has infinite index in $G$. 
Proof. If every centraliser had finite index, then by intersecting all these centralisers, we discover that the centre of $G$ has finite index in $G$; whence since $G$ is infinite, there is a free abelian subgroup of finite index.

Lemma 3.6. Suppose that $W$ is a Coxeter group for which the product of reflections $s \cdot t$ has infinite order. Then either $W$ is virtually $\mathbb{Z}$ or there is a surjection $\pi: W \rightarrow W^{\prime}$, where $W^{\prime}$ is an infinite Coxeter group and $\pi(s) \cdot \pi(t)$ has finite order.

Proof. Adding the relation $(s \cdot t)^{7}=1$ gives a Coxeter group $W^{\prime}$ which is a quotient of $W$. If $W^{\prime}$ is infinite we are finished. There is a Coxeter diagram for $W^{\prime}$ obtained from that for $W$ by adding an extra edge labelled 7 between the vertices for $s$ and $t$. If this Coxeter diagram of $W^{\prime}$ is connected then the classification of connected Coxeter diagrams of finite Coxeter groups ([2, p. 32]) shows that the diagram is the standard diagram for $D_{7}$, the dihedral group of order 14 . In this case $W$ has a diagram with 2 vertices and one edge labelled $\infty$, and is therefore the infinite dihedral group. Thus $W$ is virtually $\mathbb{Z}$.

Otherwise, if the Coxeter diagram for $W^{\prime}$ is not connected then $W^{\prime}$ is a direct sum of Coxeter groups, which by assumption are all finite. We may apply the above argument to the component containing the vertices labelled $s, t$.

The main theorem is true for groups which are virtually $\mathbb{Z}$. Thus to prove the main Theorem 1.1, by repeated applications of Lemma 3.6, it suffices to deal with the case that $W$ is an infinite Coxeter group all of whose two generator special subgroups are finite dihedral groups. Moreover, by Lemma 3.5, either $W$ is virtually abelian and the Theorem 1.1 follows, or else there is some reflection, $s$, with $C_{W}(s)$ of infinite index in $W$. Fix such an element $s$, and denote the unique associated wall by $\mathcal{H}$.

Recall the complex $X$ described in Section 2.3. Then $s$ acts on $X$ and its fixed set is a 1-dimensional subcomplex $X_{s}=X \cap \mathcal{H}$ of $X$. The structure of $X_{s}$ in $X$ is as follows:

Since $s$ does not fix any chamber of $\Sigma$, it follows that $s$ does not fix any vertex of $X$. If a point, $x$, on an edge, $E$, of $X$ is fixed by $s$ then $s$ exchanges the two chambers corresponding to the vertices of $E$ and thus maps $E$ linearly to itself swapping the endpoints. We claim that if $A$ is any 2-cell of $X$ which contains $E$ then $s$ maps $A$ to itself, and therefore $s \mid A$ is a linear involution. The 2-cell $A$ arises from a relation of the form $(s \cdot t)^{k}$ and corresponds to a face $\langle s, t\rangle$ of the fixed codimension-1 simplex in $\Sigma$. One checks from the form of the relation (see Figure 1) that the action of $s$ is as reflection in the associated $2 k$-gon.

Thus $X_{3}$ consists of some points in the middle of edges of $X$ together with a diameter of each 2-cell of $X$ adjacent to such an edge. In particular, it follows that each component of $X_{s}$ is uniquely determined by a single edge, $e$, in that component. One just "exponentiates" the 1-dimensional subspace determined by $e$. This means extend $e$ linearly, and on encountering an edge, $E$, of $X$ continue into each cell of $X$ adjacent to $E$ using a ray orthogonal to $E$. 

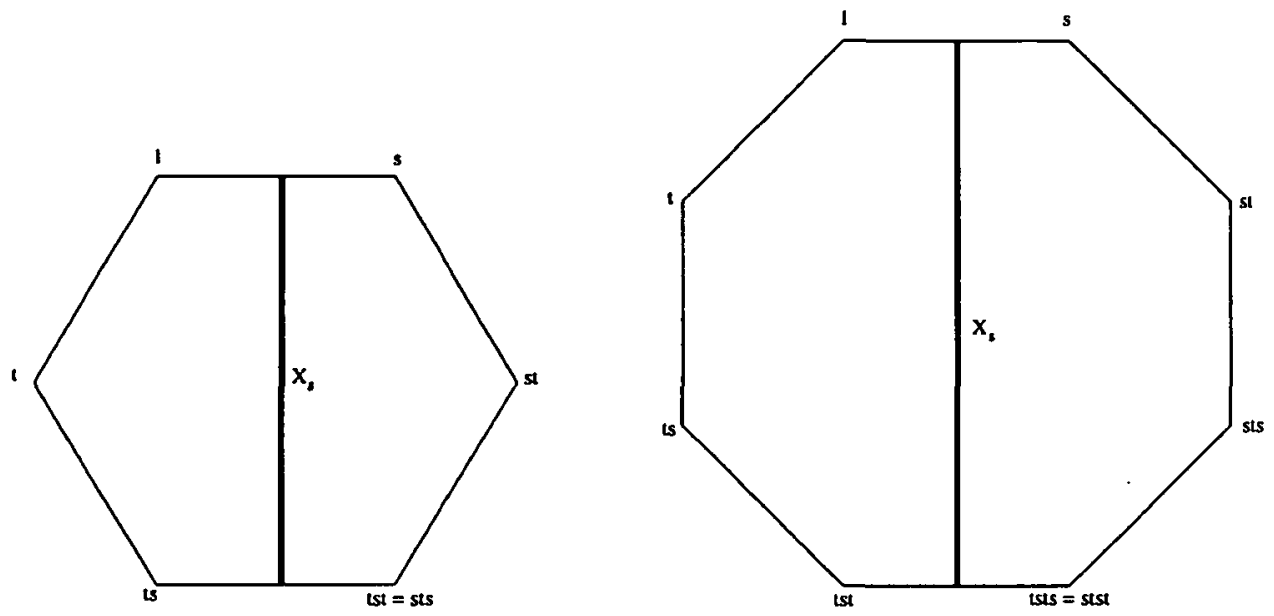

FIGURE 1

Lemma 3.7. $X_{s} / C_{W}(s)$ is a compact subcomplex of $X / C_{W}(s)$.

Proof. Note first that the argument of 2.2 shows that if $g \in W$ maps any top dimensional face of $\mathcal{H}$ to some other face of $\mathcal{H}$, then it lies in $\operatorname{stab}(\mathcal{H})$. Now $\Sigma / W$ is an $(n-1)$-simplex where $n=\operatorname{card}(S)$ thus there is a finite number, at most $n$, of $\operatorname{stab}(\mathcal{H})$ orbits of top dimensional face of $\mathcal{H}$.

From this it follows that $\mathcal{H} / C_{W}(s)$ has a finite number of top dimensional simplices. Moreover, $\mathcal{H}$ is the fixed set of a reflection so that we see from [2, Chapter III $\S 4$ Lemma 6 et seq.] that $\mathcal{H}$ is the union of its top dimensional faces, in particular, $\mathcal{H} / C_{W}(s)$ is compact.

Thus $X_{s} / C_{W}(s)$ is a closed subcomplex of the compact set $\mathcal{H} / C_{W}(s)$, which implies the result.

Proof of Theorem 1.1. Our methods are motivated by considerations coming from low-dimensional topology. We call a subcomplex, $K, 2$-sided in a complex $X$ if $K$ has a neighbourhood $K \times[-1,1]$ with $K$ identified with $K \times 0$. The proof proceeds by finding a (torsion-free) subgroup, $F^{(3)}$, of finite index in $W$ such that $X / F^{(3)}$ contains a compact, non-separating, 2-sided, 1-subcomplex $K$. There is a map of $X / F^{(3)}$ to the circle given by mapping the complement of $K \times[-1,1]$ to a point and mapping $K \times[-1,1]$ on to $[-1,1]$ which is then wrapped round the circle. Thus $H^{1}\left(X / F^{(3)}, \mathbb{Z}\right)$ is non-zero. Hence $F^{(3)}$ maps onto $\mathbb{Z}$.

Let $q: X \rightarrow X / C_{W}(s)$. By Lemma 3.7, $q\left(X_{s}\right)$ is a compact subcomplex of $X / C_{W}(s)$. We claim that if two translates $q\left(w \cdot X_{s}\right)$ and $q\left(w^{\prime} \cdot X_{s}\right)$ meet in an edge in $X / C_{W}(s)$, then they actually coincide. The reason is that this implies that there is an element $c \in C_{W}(s)$ so that in $X, w \cdot X$, meets $c \cdot w^{\prime} \cdot X$, in an edge which in turn means that the 
walls $w \cdot \mathcal{H}_{s}$ and $c \cdot w^{\prime} \cdot \mathcal{H}_{s}$ meet in a codimension 1 simplex of the Coxeter complex; however two walls which meet in this way coincide. Thus $w \cdot X_{s}$ coincides with $c \cdot w^{\prime} \cdot X_{s}$, so that $q\left(w \cdot X_{s}\right)=q\left(w^{\prime} \cdot X_{s}\right)$. This happens if and only if $w$ and $w^{\prime}$ determine the same double coset of $C_{W}(s)$.

Since $X$ is locally finite, it follows that $X / C_{W}(s)$ is also locally finite. It now follows that there are only finitely many distinct sets $q\left(w \cdot X_{s}\right)$ which can intersect $q\left(X_{s}\right)$.

To see this, note that any such intersection occurs in the 2-cells of $X$ and appears as an arc of $q\left(X_{s}\right)$ crossing an arc of $q\left(w \cdot X_{s}\right)$. This intersection is transverse since the above paragraph shows the intersection cannot be along an edge. The same argument shows that arcs of $q\left(w \cdot X_{s}\right)$ cannot coincide for different $w$ 's. Each edge $e$ of $q\left(X_{s}\right)$ lies in some 2-cell and these arguments show that only a finite number of sets of the form $q\left(w \cdot X_{s}\right)$ can cross $e$. The set $q\left(X_{s}\right)$ is compact, so that it has only a finite number of edges, proving the claim.

Let $\left\{w_{1}, \ldots, w_{p}\right\}$ be a set of representatives. By Corollary 3.2 , we may find a subgroup $F$ of finite index in $W$ which contains $C_{W}(s)$ and excludes all the elements $\left\{w_{1}, \ldots, w_{p}\right\}$. It follows that $F$ contains no element in any of the double cosets $C_{W}(s) w_{i} C_{W}(s)$. Thus when $F$ acts on $X, X_{s}$ meets none of its translates.

Since $W$ is a linear group, there is a normal torsion-free subgroup, $T \triangleleft W$, of finite index in $W$. Then the quotient map $p_{T}: X \rightarrow X / T$ is a covering space since the stabiliser in $W$ of a cell of $X$ is finite. It will also be useful to consider the subgroup $W_{+}$, of index 2 in $W$ which is the kernel of the map $W \rightarrow \mathbb{Z}_{2}$ given by sending each generator in $S$ to $1 \in \mathbb{Z}_{2}$.

Set $F^{\prime}=F \cap T \cap W^{+}$and $K=C_{W}(s) \cap F^{\prime}$.

Lemma 3.8. The complex $X_{s} / K$ is 2-sided in $X / F^{\prime}$.

Proof. The complex $\Sigma / W_{2}$ is the double of an $n-1$ simplex; label the two chambers + and - ; this gives rise to a labelling of the chambers in $\Sigma$ which is preserved by the action of the group $W^{+}$, hence by the group $F^{\prime}$. This gives a way to assign a local product neighbourhood to a wall for which the positive and negative sides are preserved by the group $F^{\prime}$.

This two-sidedness in the Coxeter complex then implies a two-sidedness for walls in $X$ which is preserved by $F^{\prime}$ and the lemma follows.

If $X_{s} / K$ is not connected, we replace it by one of its connected components. If $X_{s} / K$ does not separate $X / F^{\prime}$ we are finished. If $X_{s} / K$ does separate $X / F^{\prime}$, we argue as follows. Firstly note that if we set $A_{ \pm}$to be the components of the complement of $X_{s} / K$, then neither of the inclusion maps

$$
i_{ \pm}: \pi_{1}\left(X_{s} / K\right) \cong K \rightarrow \pi_{1}\left(A_{ \pm}\right)
$$

can surject, for application of the reflection $s$ shows that a surjection on one side implies a surjection on both sides, so that $K=F^{\prime}$ would have finite index in $W$, contradicting our choice of $s$. Choose elements $a_{ \pm}$which lie in $\pi_{1}\left(A_{ \pm}\right)-i_{ \pm}(K)$. 
By standard arguments, $K$ is separable inside $F^{\prime}$, so that we may find a subgroup $F^{\prime \prime}$ of finite index in $F^{\prime}$ which contains $K$ and excludes both the elements $a_{ \pm}$. Intersecting $F^{\prime \prime}$ with all its $W$ conjugates defines a regular covering $r: X / F^{(3)} \rightarrow X / F^{\prime}$. Although it is not in general true that $K \leq F^{(3)}$, we claim that a connected component of the preimage of $r^{-1}\left(X_{s} / K\right) \subset X_{s} / F^{(3)}$ does not separate $X / F^{(3)}$.

To see this we argue as follows: Define a bipartite graph $\Gamma=\Gamma\left(F^{(3)}\right)$ in $X_{s} / F^{(3)}$ by taking a vertex for each connected component of $r^{-1}\left(A_{ \pm}\right)$and connect two vertices by one edge for each component of $r^{-1}\left(X_{s} / K\right)$ along which they are adjacent. The covering is connected, whence so is $\Gamma$. Moreover, the intermediate covering $X / F^{\prime \prime} \rightarrow X / F^{\prime}$ has the property that if we form the analogous complex $\Gamma\left(F^{\prime \prime}\right)$, this has at least one vertex on each side with valence at least 2 . This guarantees that the graph $\Gamma$ of the regular covering corresponding to $F^{(3)}$ has no vertex of valence one, so that it cannot be a tree. The obvious retraction mapping $\rho: X / F^{(3)} \rightarrow \Gamma$ exhibits the required homology in the subgroup $F^{(3)}$.

Remarks. Continuing the theme of the introduction where we discussed properties of Coxeter groups similar to those of lattices in Lie groups. For Coxeter groups which are not virtually abelian, the existence of a map to $\mathbb{Z}$ suggests that in some sense this class of Coxeter groups cannot have the Congruence Subgroup Property (cf. [1]).

It is also worth noting that from a more algebraic perspective our proof shows (in the terminology of Scott) that there is a subgroup $H$ of finite index in $C_{W}(s)$, with the pair $(W, H)$ having at least two ends. Given this observation the final part of the proof could be bypassed by using the somewhat more general:

Theorem 3.9. [11]. Let $G$ be an almost finitely presented group. Then $G$ splits as an $H N N$ extension over some finitely presented subgroup if and only if it has a separable subgroup $H$ with $e(G, H) \geq 2$.

\section{REFERENCES}

1. H. Bass, J. Milnor and J-P. Serre, Solution to the Congruence Subgroup Problem for $\mathrm{SL}_{\mathrm{n}}(n \geq 3)$ and $\mathrm{Sp}_{2 \mathrm{n}}(n \geq 2)$, Publ. I. H. E. S. 33 (1967), 421-499.

2. K. Brown, Buildings (Springer-Verlag, 1989).

3. M. Bozejko, T. Januszkiewicz and R. Spatzier, Infinite Coxeter groups do not have Kazhdan's property. J. Operator Theory 19 (1988), 63-68.

4. M. DAvis, The cohomology of a Coxeter group with group ring coefficients, preprint.

5. P. DE LA HARPE and A. VAletre, La propriété ( $T$ ) de Kazhdan pour les groupes localement compacts, Astérisque 175 (1989).

6. C. Gonciulea, Infinite Coxeter groups virtually surject onto $\mathbb{Z}$, Comment. Math. Helv. 72 (1997), 257-265.

7. A. HaEfliger, Complexes of Groups and Orbihedra, in Group Theory from a Geometrical Viewpoint (World Scientific, 1991).

8. J. Hempel, Orientation reversing involutions and the first betti number of finite coverings of 3-manifolds, Invent. Math. 67 (1982), 133-142. 
9. D. D. LoNG, Immersions and embeddings of totally geodesic surfaces, Bull. London Math. Soc. 19 (1987), $481-484$.

10. D. D. LoNG and G. NiBLo, Subgroup separability and 3-manifold groups, Math. Z. 207 (1991), 209-215.

11. G. NibLo, Finding splittings of groups and three manifolds, Bull. London Math. Soc. 27 (1995), 567-574.

12. G. A. MARGUlis, Discrete Subgroups of Semi-simple Lie Groups (Ergebnisse der Mathematik und ihr Grenzgebeite, Springer-Verlag, 1991).

COOPER AND LONG

Department of Mathematics

UNIVERSITY OF CALIFORNIA

Santa Barbara, CA 93106

U.S.A.
REID

Department of Mathematics

UNIVERSITY OF TEXAS

AUstin, TX 78712

U.S.A. 